\title{
Enhancing Text Readability in Damaged Documents
}

\author{
Gideon Frieder
}

Documents can be damaged for various reasons - attempts to destroy the document, aging, natural cause such as floods, etc. In the preliminary work reported herein, we present some results of processes that enhance the visibility of lines, therefore the readability of text in such documents. No attempt is made to interpret the contents rather, the work intends to aid an analyst that will eventually process the information that is now easier to see and acquire.

Our software provides different methods of enhancement, and the analyst can choose the method and set the optimal parameters. The results that we have are rather encouraging, however, the processing is done on every page separately, and the choice of parameters to achieve optimal enhancement is difficult. The major obstacle for automating this process and providing a tool which can be used for a reasonable - even massive - amount of data lies in the absence of a mathematical definition of readability, and ability to create a figure of merit whose value will guide the automatic selection of optimal parameters. We shall present our results, and hope that it will motivate the listeners to help in finding such figure of merit.

The corpus of data used in this presentation consists of the diaries of HaRav Dr. Avraham Abba Frieder. These diaries consist of more then 800 pages written in different scripts, both typed and handwritten, in different languages, in different states of readability; with intermix of text, pictures, tables, corrections etc.

The diaries are now on a permanent loan to the archives of Yad Va Shem, and can be found on the net in www.ir.iit.edu/collections

This work was initiated while the presenter was visiting the imaging laboratory of the University of Bremen. It was continued and greatly expanded by Dr. Gady Agam and his collaborators in the IR@IIT Laboratory, under the direction of Dr. Ophir Frieder. 